\title{
Impact of Crossover Probability on Symmetric Travel Salesman Problem Efficiency
}

\author{
http://dx.doi.org/10.3991/ijim.v9i1.4196
}

\author{
S.F.Abu-owida and W.S.Alsharafat \\ Al al-Bayt University, Mafraq, Jordan
}

\begin{abstract}
Genetic algorithm (GA) is a powerful evolutionary searching technique that is used successfully to solve and optimize problems in different research areas. Genetic Algorithm (GA) considered as one of optimization methods used to solve Travel salesman Problem (TSP). The feasibility of GA in finding TSP solution is dependent on GA operators; encoding method, population size, number of generations in general. In specific, crossover and its probability play a significant role in finding possible solution for Symmetric TSP (STSP). In addition, crossover should be determined and enhanced in term reaching optimal or at least near optimal. In This paper, we spot the light on using modified crossover method called Modified sequential constructive crossover and its impact on reaching optimal solution. To justify the relevance of parameters value in solving TSP, a set comparative analysis conducted on different crossover methods values.
\end{abstract}

Index Terms-Genetic Algorithm, Crossover, mutation, TSP.

\section{INTRODUCTION}

The Travelling Salesman Problem (TSP) is a relatively ancient problem: it was precept as forward as 1759 by Euler, who's interest was in solution the Knights' tour proposition [1] .The extremity 'move seller' was first usage in 1932, in a German Leger 'The travelling salesman, how and what he should do to get mandate and be fruitful in his businesses, literal by a veteran journey salesman[2].The origins of the TSP in mathematics aren't actually understood -all we know for certain is that it occurs around 1931 by the mathematicians Sir William Rowaw Hamilton and Thomas Penyngton Kirkman from Ireland and Britain relatively [2].

The TSP is one of hard and old problems in computer science. It can be stated as: a graph with vertex (cities), and edge (distance, or travel time etc.). The TSP implemented to find shortest tour that each city visited exactly once and then returns to the starting city.

The TSP is categorized in two groups: symmetric travelling salesman problem (STSP) where the distances between two cities equal forward and backward and there are $(n-1) ! / 2$ possible solutions. In opposite, Asymmetric TSP considered the distances between two cities where forward path differ than backward path and there are (n $-1)$ ! possible solutions.[3]

In a STSP is complete undirected graph $\mathrm{G}=(\mathrm{V}, \mathrm{A})$ where $V=\{1, \ldots, n\}$ is a cites set, $E=\{(i, j): i, j \in V, i<j\}$ is an edge set and $\{(\mathrm{i}, \mathrm{j}): \mathrm{i}, \mathrm{j} \in \mathrm{V}, \mathrm{i} \neq \mathrm{j}$ is an arc set. A cost matrix $\mathrm{C}$ (cii) is defined on $\mathrm{E}$ or on $\mathrm{A}$. The cost matrix satisfies the triangle inequality whenever $\operatorname{cij} \leq \mathrm{Ci} \mathrm{k}+\mathrm{C} \mathrm{k} \mathrm{j}$, for all $\mathrm{i}, \mathrm{j}, \mathrm{k}$. In particular, this is the case of a planar problem for which the vertices are points $\mathrm{pi}=(\mathrm{xi}, \mathrm{yi})$ in the plane, and $\mathrm{cij}=\sqrt{\left(x_{i}-x_{j}\right)^{2}+\left(y_{i}-y_{j}\right)^{2}}$ is the Euclidean distance. The triangle inequality is also satisfied if cii is the length of the shortest path $i$ from $j$ to on $G[4]$.

\section{RELATED WORK}

In term of finding optimal solution for STSP, if possible, several methods have been adapted and enhanced to reach this target. In addition, several researches have worked on modifying crossover techniques which considered as one of Genetic Algorithm (GA) operations.

Ahmed in [5] has adapted a new crossover operator called Sequential Constructive crossover (SCX). SCX works by producing a child by using better parents edges based on their values that may exist in structure of parents and maintain the node sequence as in parent. After that, If the node not exists in any of the parent, then perform search within the nodes $\{2,3, \ldots, n\}$ to find the node.

Whitley in [6] has adapted Hybrid Genetic Algorithm for solving TSP called Generalized Partition Crossover (GPX) .The GPX is a hybrid GAs with LK-search which derived from Partition Crossover (PX). LK-search works by finding better tours using double bridge moves. The GPX find near optimal in responsible time.

Researchers in [7] have focused on cyclic crossover (CX) the comparative study and analysis of the Partially Matched Crossover (PMX), and ordered crossover (OX) operators. In this paper used Roulette Wheel to selected parents, 1000 iterations as terminate conditions and randomly mutations used in this paper. The results show that the distance measured by PMX the minimum as compared to distance measured by $\mathrm{CX}$ operator and distance measured by $\mathrm{CX}$ operator is less than the distance measured OX that mean PMX crossover gain improvement when comparative with the $\mathrm{CX}$ and $\mathrm{OX}$ crossover operator.

Researchers in [8] have proposed Greedy Crossover called Improved Greedy Crossover (IGX) to solve STSP using GAs. IGX search for one node using two auxiliary double-linked lists that considered as a resource for two parents to reduce memory usage. When a node is selected to be part child structure, offspring, it will be deleted from both double-liked. So IGX targeted to select the offspring depended on closer to their parents.

Ahmed in [9] has proposed the ordered clustered travelling salesman problem. This method focused on divided vertex network into predetermined clusters to find the least cost for TSP. In this paper companies a hybrid genetic algorithm using sequential constructive crossover, 2 option search, and a local search. IT started with vertex 1 and sequentially searches both of the parent chromosomes 
and considers the first unvisited vertex of the present cluster appearing after vertex 1 in each parent. If no unvisited vertex after vertex 1 is available in any of the parents, search sequentially from the starting of that parent and consider the first unvisited vertex of the cluster. Secondly, selected the node closely to selected node If there is not any vertex left in that cluster, then go to the next cluster. Finally, if the offspring is a complete chromosome, then stop; otherwise, rename the present vertex.

Patvichaichod and his colleagues in [10] have developed the new procedures of GAs, which is called Hybrid Encoding GAs with multi-relations, HEGAs. The HEGAs is developed by using a new encoding, which mixes the binary encoding with the integer encoding using GAs for investigate TSP problem which each of the vertices has many edges and different cost. The methods worry from the different weights in which the problem has more complexity. The algorithm representation method of chromosomes using binary (represents the edges) and integer numbers (represents the vertices). In the HEGAs, the one-point crossover is used and in the HEGA, the exchange mutation is used by sampling the two genes from the vertices and the values are exchanged .Finally, the next evolution to a new generation is then begun and the evolution progress persevere to the determine generation, which is the termination of the HEGAs.

Different optimization techniques have been adapted to solve TSP. A number of these methods are; Simulating Annealing in [11], Swarm Intelligent [12], Ant colony [13], Neural network [13, 14] beside of Genetic Algorithm.

\section{GenetiC Algorith With Modified CROSSOVER}

GA is an optimization problem has been adapted in term of finding solution for complex problem as TSP. To implement GA, a set of operations take a place to gain results starting from encoding routes to construct population. All populations are evaluated using fitness function. Next new generation will be produce by implementing selection, crossover and mutation taking into account termination criteria to determine whether continue to produce next generation or stop. The main GA operation as follows:

\section{A. Route encoding}

There are several encoding method can be used such as binary, real and integer. For solving TSP, integer coding has been selected to represent cities. Where each cities position can take any integer in $\{1,2 \ldots n\}$ without any duplication of cities. Figure 1; represent an example on route encoding.

router $1:\{5->16->6->1->10->9->3->17->8->15->12-$ $>4->13->14->2->7->11\}$.

\section{Figure 1. Route encoding}

\section{B. Fitness function}

The GA used quantifies function called fitness function which acts as indicator for reaching solution. This value calculated for each individual in population. In TSP, fitness function represents calculated distance between cities. The object function for TSP is minimizing fitness value as represented in following equation 4.1.
Where $f(x)$ is calculated cost of the tour represented by route.

\section{Selection}

To maintain the diversity between generations, selection parents that they will participate in producing new childs, offspring. For this purpose, different selection methods have been developed by researchers to accomplish this task. In this work we adapted Roulette Wheel Selection (RWS). RWS focused on selecting parents with high probability where this probability depends on parent's fitness value. RWS works by adding all individual fitness value (denoted by $\sum$ fi) then calculate the expected probability for each individual by dividing its fitness value over cumulative fitness values for all parents as shown in equation 2.

$$
\mathrm{e}_{\mathrm{i}}=\frac{\mathrm{F}_{\mathrm{i}}}{\overline{\mathrm{F}}}
$$

(2) Where:

Fi: Individual fitness
F: Average fitness

\section{Modified Sequential Constructive Crossover (MSCX)}

We have developed crossover operator in term of reaching optimal solution of TSP. This operator gives results which are remarkably better than the others crossover operator .It produces a child, offspring, by taking advantage of better links values provided by their parents. It also uses the better links value, which are not present in the parent's structure. The detailed clarifications about how it works are as follows:

1. Start

2. Parents split into group.

3. Start from initial node presented in parent 1 .

4. Sequentially search in all groups in the both of the parents and take care into unvisited node that appeared after the node 1 in both parents. If don't found the next node (the node that is not yet visited) after initial node in both parents, search in data set to find minimum cost. After this go to (5).

5. Suppose the 'node $\alpha$ found in parent1 while node $\beta$ ' found in parent 2 , then selecting the next node and go to (7).

6. If cost of $\alpha<\operatorname{cost}$ of $\beta$, then select node $\alpha$, otherwise select node $\beta$ as the next node and considered as a part of a child, offspring, If the offspring is a complete, then stop, otherwise, return to (4).

\section{Repeat}

\section{E. Mutation}

Mutation takes a place after crossover with certain probability denoted by $\mathrm{P}_{\mathrm{m}}$. Mutation is random changing in cities order in route for produced Childs. Mutation concerns in introducing certain diversity in the population to be differ than existing population. In addition, mutation suitable for more exploration to discover unknown situations in search space and avoid local optima. For experimental tests $\mathrm{P}_{\mathrm{m}}=0.01$. 


\section{F. Termination}

Different termination criteria may take a place to stop. One of termination criteria is running the algorithm according to specific number of iterations, or when fitness value converges to stable value. In this paper we considered number of iterations as stopping criteria which equals 10000 iterations.

\section{EXPERIMENTAL RESULTS}

In this section, we investigate the effectiveness of modifying crossover operation in GA. For comparison purposes, we used Error of solution for each instance and average as a measurement tool. In addition to perform experimental result we use benchmark STSP instance reported in TSPLIB [16]. Table I presents the main features for dataset instances that will be used in comparison.

TABLE I.

INSTANCES PROPERTIES

\begin{tabular}{|c|c|c|}
\hline Instances & Number of cites & Optimal \\
\hline gr17 & 17 & 2085 \\
\hline fri26 & 26 & 937 \\
\hline bays29 & 29 & 2020 \\
\hline dantzig42 & 42 & 699 \\
\hline swiss42 & 42 & 1273 \\
\hline
\end{tabular}

In Table II, we notice that MSCX achieves the intended target to reach near optimal solution measured by low error rate.Figure 1 shows these results.

TABLE II.

TABLE 2: MSCX ERROR \%

\begin{tabular}{|c|c|}
\hline Instances & Error \% \\
\hline gr17 & 0.00 \\
\hline fri26 & 0.00 \\
\hline bays29 & 0.01 \\
\hline dantzig42 & 0.04 \\
\hline swiss42 & 0.03 \\
\hline
\end{tabular}

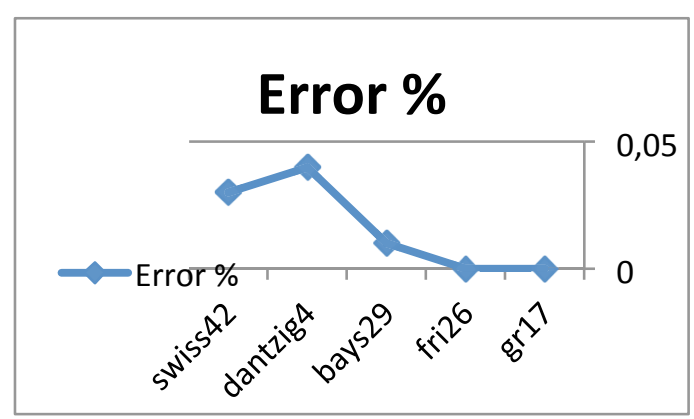

Figure 2. MSCX error rate

For comparative purposes, we compare MSCX results with HGA because both HGA and MSCX used STSP dataset instances.

In Table III, we present comparative between HGA and MSCX which applied on different city size. This shows that MSCX gained approximately better result than HGA. Figure 2, presents result in table 3 .
TABLE III.

ERROR \% FOR HGA AND MSCX

\begin{tabular}{|c|c|c|}
\hline \multirow{2}{*}{ Instances } & HGA & MSCX \\
\cline { 2 - 3 } & Error \% & Error \% \\
\hline gr17 & 0.21 & 0 \\
\hline fri26 & 0.02 & 0 \\
\hline dantzig42 & 0 & 0.04 \\
\hline swiss42 & 0.26 & 0.03 \\
\hline
\end{tabular}

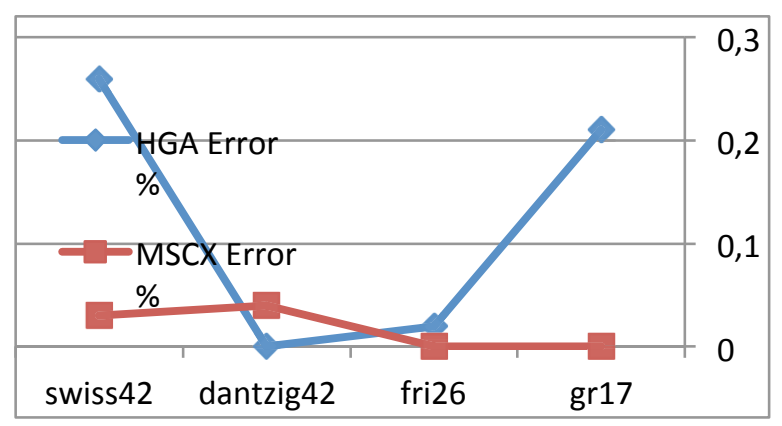

Figure 3. Error \% for HGA and MSCX crossover.

\section{CONCLUSION AND FutURE WORK}

In this paper, we provide modified crossover operation, MSCX, which shows improvement in solving TSP by tuning up GA operations as crossover. In addition, GA considered an efficient algorithm in solving optimization problems as TSP. To achieve better results more investigations have to be taken on crossover probability and how the results will be affected by changing type of GA.

\section{REFERENCES}

[1] Matai1, R., S.P, Singh and Matai. M. 2010. Traveling Salesman Problem: An Overview of Applications, Formulations, and Solution Approaches. In Tech.www.intechopen.com.

[2] Michalewicz, Z. (1994), Genetic Algorithms + Data Structures = Evolution Programs, (2nd ed), Berlin: Spring.

[3] Davendra, D. (2010), Traveling Salesman Problem, Theory and Applications, (1st ed), Croatia: InTech. http://dx.doi.org/10. $\underline{5772 / 547}$

[4] Gupta, S., Panwar. P, (2013), Solving Travelling Salesman Problem Using Genetic Algorithm, International Journal of Advanced Research in Computer Science and Software Engineering, Accepted for Publication.

[5] Ahmed.Z. (2010), Genetic Algorithm for the Traveling Salesman Problem using Sequential Constructive Crossover Operator, International Journal of Biometrics \& Bioinformatics, Accepted for Publication.

[6] Whitley D, Hains. D and H. Adele. (2010), A Hybrid Genetic Algorithm for the Traveling Salesman Problem using Generalized Partition Crossover, Accepted for Publication.

[7] Kumar .N, Karambir, Kumar.R, (2012), A Comparative Analysis of PMX, CX and OX Crossover operators for solving Travelling Salesman Problem, International Journal of Latest Research in Science and Technology, Accepted for Publication.

[8] Esmkhan $\mathrm{H}$ and Zamanifar.K, (2012), Developing Improved Greedy Crossover to Solve Symmetric Traveling Salesman Problem, Accepted for Publication.

[9] Ahmed.Z, (2014), the Ordered Clustered Travelling Salesman Problem: A Hybrid Genetic Algorithm, Hindawi, Accepted for Publication Programs, (2nd ed), Berlin: Spring

[10] Patvichai chod, S., (2011). An Improved Genetic Algorithm for the TravelingSalesman Problem with Multi-Relations, Journal of Computer Science, Accepted for Publication. 


\section{SHORT PAPER}

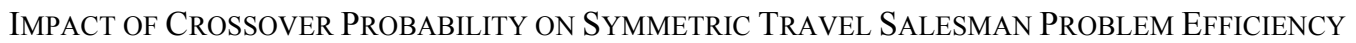

[11] Genga,X., Chenb,Z., Yanga,W., Shia,K., and Zhaoa, K. 2011. Solving the traveling salesman problem based on an adaptive simulated annealing algorithm with greedy search, Applied Soft Computing, Volume 11, Issue 4, June 2011, Pages 3680-3689. http://dx.doi.org/10.1016/j.asoc.2011.01.039

[12] Tao, Y., Cui, D., Miao, X., and Chen, H. 2007. An Improved Swarm Intelligence Algorithm for Solving TSP Problem. Third International Conference on Intelligent Computing, ICIC 2007, Qingdao, China, August 21-24.

[13] Su,Z., Hlaing and Khine,A. 2011. Solving Traveling Salesman Problem by Using Improved Ant Colony Optimization Algorithm, , IACSIT, International Journal of Information and Education Technology, Vol. 1, No. 5, December 2011.

[14] Siqueira,P., Scheer,S., and Steiner, M. 2008. A Recurrent Neural Network to Traveling Salesman Problem. Travelling Salesman
Problem, ISBN 978-953-7619-10-7, pp. 202, September 2008, ITech, Vienna, Austria.

[15] Reilly, R., Tichmev,P. 2003. Neural network approach to solving the Traveling Salesman Problem, Journal of Computing Sciences in Colleges, Volume 19 Issue 1, October 2003, Pages 41-61.

[16] http://comopt.ifi.uni-heidelberg.de/software/TSPLIB95/

\section{AUTHORS}

S.F.Abu-owida and W.S.Alsharafat are with Prince Hussein Bin Abdullah Faculty of Information Technology, Al al-Bayt University, Mafraq, Jordan.

Submitted 08 October 2014. Published as resubmitted by the authors 25 January 2015. 